\title{
RASSF8 downregulation promotes lymphangiogenesis and metastasis in esophageal squamous cell carcinoma
}

\author{
Lan Zhang ${ }^{1,3,4, *}$, Jian-Hua Wang ${ }^{5, *}$, Rong-Xin Liang ${ }^{5, *}$, Shu-Ting Huang ${ }^{1,3,4}$, Jing \\ $\mathbf{X u}^{3,4}$, Lin-Jing Yuan ${ }^{1,3,4}$, Long Huang ${ }^{6}$, Yun Zhou ${ }^{1,3,4}$, Xing-Juan Yu ${ }^{3,4}$, Shao-Yun \\ Wu ${ }^{3,4}$, Rong-Zhen Luo ${ }^{2,4}$, Jing-Ping Yun ${ }^{1,2,3}$, Wei-Hua Jia ${ }^{3,4}$ and Min Zheng ${ }^{1,3,4}$ \\ ${ }^{1}$ Department of Gynecology, Guangzhou, P. R. China \\ 2 Department of Pathology, Sun Yat-Sen University Cancer Center; Guangzhou, P. R. China \\ ${ }^{3}$ State Key Laboratory of Oncology in South China, Guangzhou, P. R. China \\ ${ }^{4}$ Collaborative Innovation Center for Cancer Medicine, Guangzhou, P. R. China \\ ${ }^{5}$ Cardiovascular Department, Second People's Hospital of Guangdong Province, Guangzhou, P. R. China \\ ${ }^{6}$ Department of Oncology, The Second Affiliated Hospital, Nanchang University, Nanchang, P. R. China \\ * These authors have contributed equally to this work \\ Correspondence to: Min Zheng, email: zhengmin@sysucc.org.cn
}

Jian-Hua Wang, email: wangjh296@163.com

Keywords: tumor metastasis; RASSF8; lymphangiogenesis; esophageal cancer

Received: July 08, $2015 \quad$ Accepted: September 05, $2015 \quad$ Published: September 30, 2015

This is an open-access article distributed under the terms of the Creative Commons Attribution License, which permits unrestricted use, distribution, and reproduction in any medium, provided the original author and source are credited.

\section{ABSTRACT}

Lymphatic vessels are the major routes of human esophageal squamous cell carcinoma (ESCC) metastasis. Tumor cells secrete pro-lymphangiogenic factors to induce new lymphatic vessels, promoting lymph node metastasis. In this study, we show that RAS association domain family 8 (RASSF8) expression in ESCC clinical samples was inversely correlated with lymph node metastasis and patients survival. Tumor cells with low RASSF8 expression had higher apparent migratory ability, and promoted and lymphangiogenesis both in vitro and in vivo. RASSF8 downregulation enhanced VEGF-C expression and caused subcellular redistribution of p65 in ESCC. Our results show that RASSF8 acts as a tumor suppressor in ESCC and is a potential therapeutic target for preventing lymph node metastasis.

\section{INTRODUCTION}

Human esophageal squamous cell carcinoma (ESCC) is one of the most lethal malignancies worldwide; it ranks fourth among the top 10 cancer-related deaths in China $[1,2]$. Despite advances in diagnosis and treatment over past decades, the clinical outcome of ESCC remains poor due to aggressive malignancy and rapid progression. The presence of cancer cells in the lymph nodes is one of the main negative prognostic factors of ESCC [3]. However, the mechanism by which cancer cells reach the lymph nodes remains poorly understood.

Lymphatic vessels are the major routes of tumor metastasis in ESCC. There is accumulating evidence that, despite the preexisting lymphatic vessels, tumor-associated lymphangiogenesis is an important feature of tumor progression and may facilitate cancer cell dissemination to the lymph nodes $[4,5]$. Accordingly, numerous clinical studies have demonstrated a significant correlation between lymphatic vessel density and lymph node metastasis. Clinical evidence suggests that the vascular endothelial growth factor (VEGF) family members VEGF-C and VEGF-D are major lymphangiogenic regulators [6]. Higher VEGF-C expression in ESCC is associated with higher peritumoral lymphatic vessel density, increased lymphatic invasion, and increased lymph node metastasis [7, 8]. VEGF-C and VEGF-D stimulate lymphatic vessel sprouting via VEGF receptor (VEGFR)-3 binding, which is expressed in lymphatic endothelial cells to activate downstream extracellular signal-regulated kinase (ERK) and AKT signaling [9]. Inhibiting VEGF-C/VEGFR-3 signaling suppresses tumor lymphangiogenesis and lymphatic metastasis in 
pre-clinical models and may be a promising strategy for treating ESCC and other cancers [10-13]. Besides lymphangiogenesis, the presence of cancer cells with more motility and invasiveness features also increases the risks of metastasis.

RASSF8 is one of the four new members in the RAS association domain family (RASSF). Differing from the classic RASSF members (RASSF1-6), which have a C-terminal Sav/RASSF/Hpo (SARAH) domain, RASSF7-10 contain an N-terminal RA domain and lack the SARAH domain [14-16]. RASSF8 is ubiquitously expressed in all major organs and tissues, including the brain, heart, kidney, liver, lung, and other normal adult human tissues. Endogenous RASSF8 is expressed in both the nucleus and the cell membrane, and it promotes cellcell adhesion by maintaining adherens junction stability [17]. In lung cancer, downregulated RASSF8 increases cell migration and growth, functioning as a tumor suppressor [17, 18]. However, the biological role and clinical significance of RASSF8 in ESCC remain largely unknown.

In this study, we used clinical patient samples and in vitro and in vivo models to uncover the suppressive role of RASSF8 in ESCC lymphangiogenesis and metastasis. In ESCC, RASSF8 was markedly downregulated at both mRNA and protein level, and correlated with lymph node metastasis, advanced clinical stage, and poor prognosis. RASSF8 knockdown in ESCC cells increased cell invasion ability and promoted lymphatic endothelial cell tube formation and migration; RASSF8 overexpression had the opposite effect. More importantly, downregulated RASSF8 increased VEGF-C expression by promoting the nuclear-cytoplasmic translocation of nuclear factor- $\mathrm{\kappa B}$ (NF-kB) p65, leading to lymphangiogenesis and lymphatic metastasis. These results suggest that RASSF8 is a powerful metastatic suppressor and a potential biomarker in the prognostic assessment of ESCC.

\section{RESULTS}

\section{RASSF8 downregulation correlates with poor prognosis in human ESCC}

Western blotting and quantitative real-time PCR revealed lower levels of endogenous RASSF8 in ESCC cell lines as compared with the immortalized esophageal epithelial cell line NE1 (Fig. 1A). RASSF8 mRNA levels were downregulated in eight ESCC tumor specimens as compared with the paired adjacent nontumorous tissue (ANT) samples (Fig. 1C). Consistent with this, immunohistochemical staining of 137 ESCC tissues revealed robust RASSF8 expression in the ANT epithelial components; however, RASSF8 expression was significantly downregulated in tumor tissues, particularly those with lymph node metastasis (Fig. 1B). RASSF8 protein levels were inversely correlated with clinical stage $(p=0.001$; Table 1$)$ and lymph node metastasis $(p$ $=0.002$; Table 1). Kaplan-Meier survival analysis and log-rank testing showed that patients with lower RASSF8 expression had poor clinical outcomes $(p=0.007$; Fig. 1D). Univariate and multivariate analyses indicated that low RASSF8 expression was a prognostic factor, but not an independent one, for ESCC outcome ( $p=0.009$; Table 2).

\section{ESCC lymph node metastasis correlates with downregulated RASSF8 expression at primary site}

Immunohistochemical staining of 66 lymph node metastasis-negative and 71 lymph node metastasispositive tumor tissues demonstrated significantly downregulated RASSF8 in the latter ( $p=0.004$; Fig. 1E). To determine the effect of RASSF8 on lymphangiogenesis, we investigated whether RASSF8 expression in tumors correlated with peritumoral lymphatic vessel density in ESCC. The lymphatic vessels were stained using antilymphatic vessel endothelial hyaluronan receptor (LYVE)1 antibody. As expected, decreased RASSF8 expression in ESCC was significantly correlated with increased peritumoral lymphatic vessel density in $\operatorname{ESCC}(p=0.002$; Fig. 1F). In addition, RASSF8 was downregulated in lymph node metastasis-positive samples as compared with metastasis-negative samples, such as in cervical cancer (NCBI/GEO/GSE26511; $p=0.036$; Supplementary Fig. 1A) and prostate tumor (NCBI/GEO/GSE6919; Supplementary Fig. 1B). Our results suggest that RASSF8 downregulation may facilitate lymphangiogenesis and lymph node metastasis.

\section{RASSF8 downregulation enhances ESCC cell motility and invasion in vitro}

As cell motility and invasion are important factors in cancer metastasis, we constructed RASSF8 overexpression and knockdown cell lines to characterize the effect. Efficiency was detected by quantitative real-time PCR and western blotting (Fig. 2A). RASSF8 downregulation increased ESCC cell migration speed and invasive ability as compared with the control cells $(p<0.05)$. In contrast, RASSF8 upregulation had the opposite effect ( $p<0.05$; Fig. 2B and 2C). These results suggest that RASSF8 downregulation promotes ESCC progression. 
Table 1: Correlation between RASSF8 expression and clinicopathological features in primary ESCC.

\section{RASSF8 expression}

\begin{tabular}{llll} 
Variable & Low (\%) & High (\%) & P val \\
\hline $\begin{array}{l}\text { Gender } \\
\text { female } \\
\text { male }\end{array}$ & 24 & 17 & 0.211 \\
& 45 & 51 &
\end{tabular}

Age, (years)

$<60$

39

40

0.785

$\geq 60$

30

28

Differentiation

$1-2$

3

Staging

I-II a

II b-IV

T status

$\mathrm{T} 1-\mathrm{T} 2$

T3-T4

Lymph node metastasis

No

Yes

Distant metastasis

No

60

64

0.243

Yes
17

52

16

53

24

45

26

52

42
0.962

0.002*

94

${ }^{*} p$-values indicate significance $(p<0.05)$. 
Table 2: Univariate and multivariate analyses of prognostic variables in patients with ESCC.

\begin{tabular}{|c|c|c|c|}
\hline Variable & Subset & Hazard ratio (95\% CI) & $P$ value \\
\hline \multicolumn{4}{|l|}{ Univariate analysis } \\
\hline Gender & Male versus female & $1.492(0.900-2.473)$ & 0.121 \\
\hline Age, (years) & $\geq 60$ versus $<60$ & $1.298(0.820-2.054)$ & 0.266 \\
\hline Differentiation & 3 versus $1-2$ & $1.316(1.043-1.660)$ & $0.021 *$ \\
\hline Staging & II $b-I V$ versus I-II a & $1.990(1.226-3.233)$ & $0.005^{*}$ \\
\hline T status & $\begin{array}{l}\mathrm{T} 3-\mathrm{T} 4 \text { versus } \\
\mathrm{T} 1-\mathrm{T} 2\end{array}$ & $1.874(1.027-3.421)$ & $0.041 *$ \\
\hline $\begin{array}{l}\text { Lymph node } \\
\text { metastasis }\end{array}$ & Yes versus no & $1.838(1.163-2.907)$ & $0.009 *$ \\
\hline Distant metastasis & Yes versus no & $2.571(1.266-5.208)$ & $0.009 *$ \\
\hline $\begin{array}{l}\text { Expression of } \\
\text { RASSF8 }\end{array}$ & Low versus high & $1.887(1.173-3.036)$ & $0.009 *$ \\
\hline Multivariate analy & & & \\
\hline Differentiation & 3 versus $1-2$ & $1.407(1.101-1.798)$ & $0.006 *$ \\
\hline $\mathrm{T}$ status & $\begin{array}{l}\mathrm{T} 3-\mathrm{T} 4 \\
\mathrm{~T} 1-\mathrm{T} 2\end{array}$ & $2.129(1.129-4.248)$ & 0.02 \\
\hline
\end{tabular}

CI, confidence interval. * $p$-values indicate significance $(p<0.05)$.

RASSF8 negatively regulates lymphangiogenesis in vitro and in vivo

As downregulated RASSF8 expression and lymph node metastasis of ESCC are apparently significantly correlated, it suggests that RASSF8 overexpression plays a negative role in lymphangiogenesis. To examine whether RASSF8 downregulation could stimulate tumor lymphangiogenesis, we conducted tube formation and migration assays using human lymphatic endothelial cells (HLECs). HLECs were seeded in the presence of tumor culture supernatants (TSNs) from RASSF8/RASSF8-
RNAi (RNA interference)-transfected ESCC cells. RASSF8 inhibited the ability of ESCC cells to induce tube formation and migration, whereas RASSF8 knockdown promoted it (Fig. 3A and 3B).

We evaluated the effect of RASSF8 on lymphangiogenesis and lymphatic metastasis in ESCC cells using a lymph node metastasis model. EC109 cells stably expressing RASSF8 or RASSF8-RNAi, and control EC109 cells were implanted in the footpads of BALB/cnu mice. The lymphatic vessel density in the tumors that developed from RASSF8-overexpressing cells was lower than that of tumors from the control cells. Conversely, lymphatic vessel density was significantly increased in 
tumors formed by RASSF8 knockdown cells (Fig. 4A). In addition, RASSF8 knockdown in the EC109 cells increased popliteal lymph node volume (Fig. 4B) and cytokeratin-positive immunohistochemical staining (Fig.
4C). The ratio of lymph node metastasis was decreased in the EC109-RASSF8 group and increased in the EC109RASSF8-RNAi group (Fig. 4E).

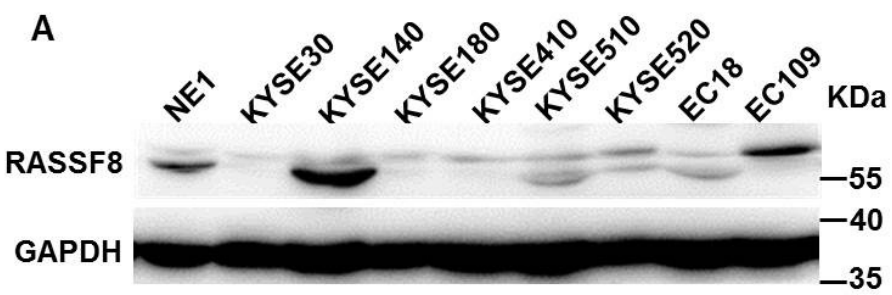

B

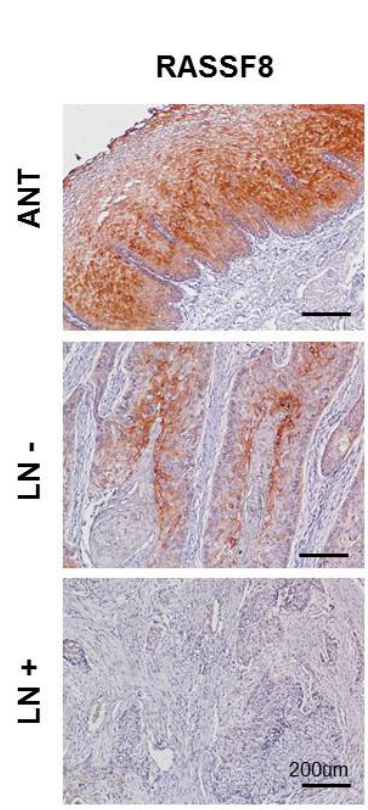

E

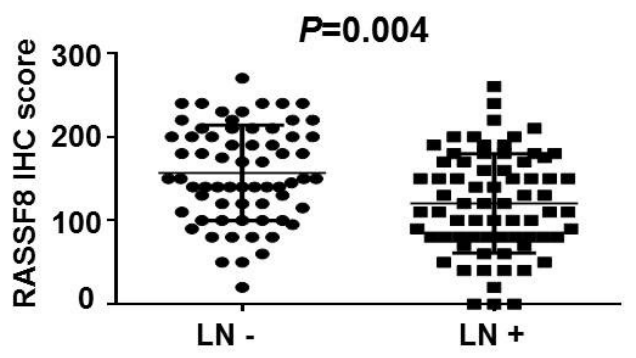

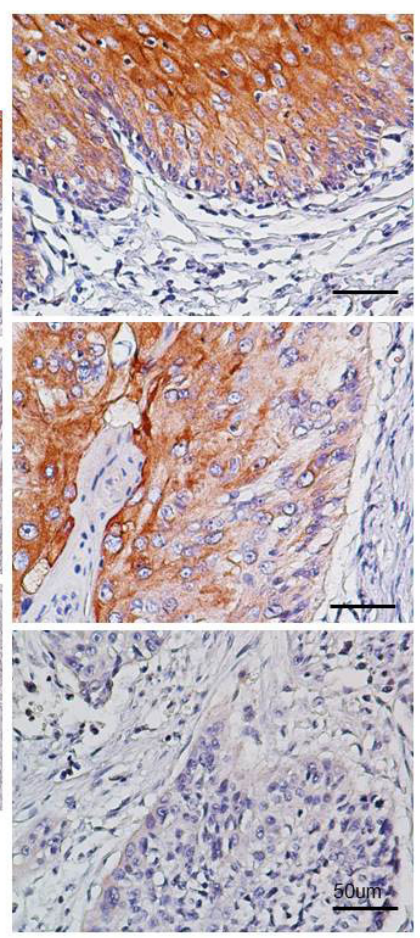

C
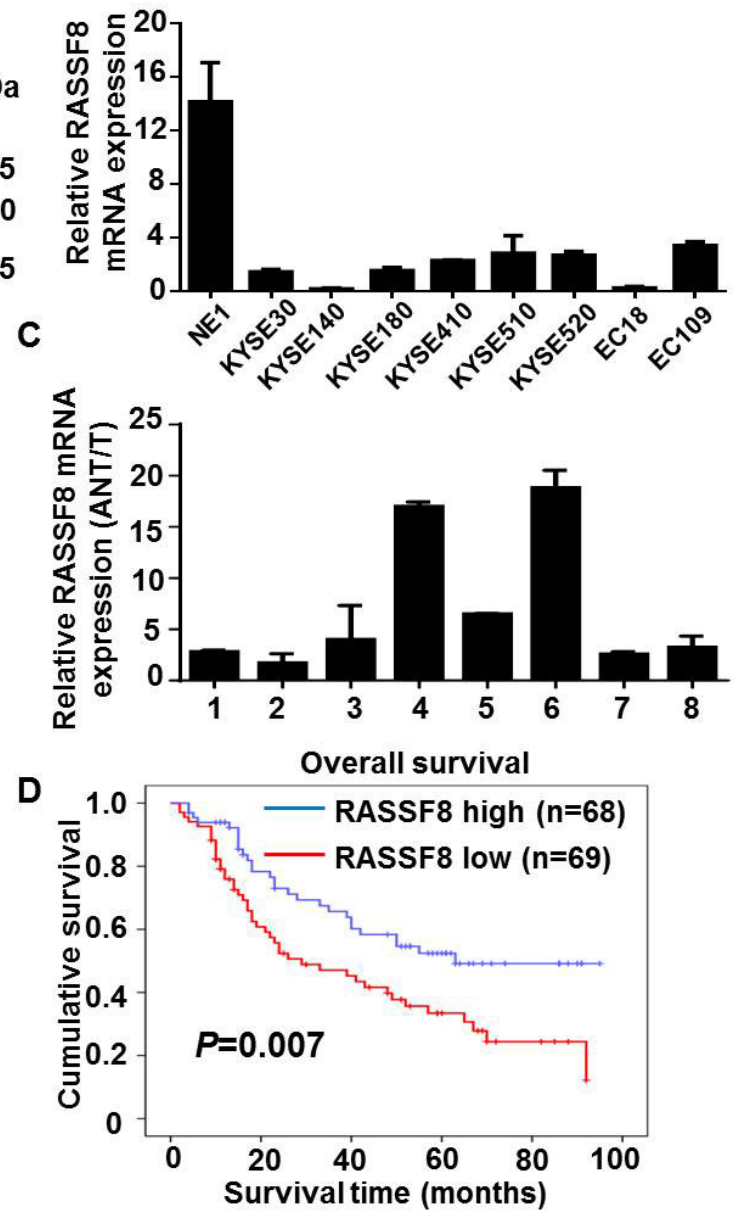

$\mathbf{F}$

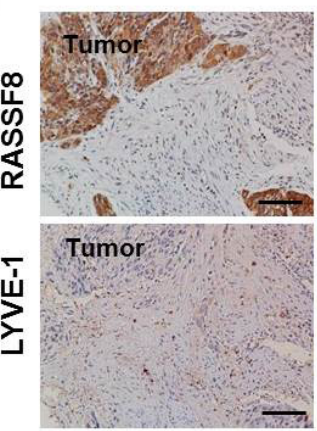

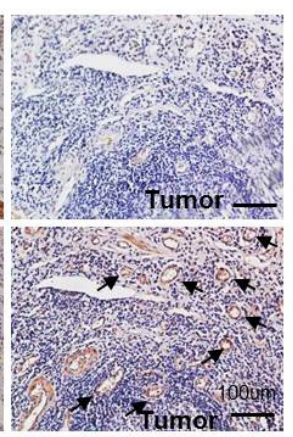

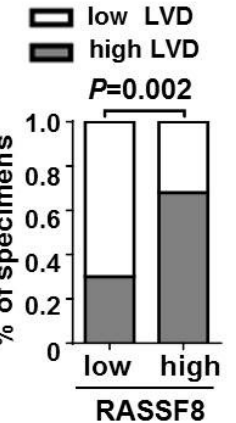

Figure 1: RASSF8 is frequently downregulated in ESCCs. A., Western blotting (left) and quantitative real-time PCR (right) results showing RASSF8 levels in eight ESCC cell lines and the NE1 immortalized esophageal epithelial cell line. B., Representative images of RASSF8 immunochemical staining in ANT and tumor tissues with or without lymph node metastasis (LN+ and LN-, respectively). Bars $=200 \mu \mathrm{m}$ (left); $50 \mu \mathrm{m}$ (right). C., Quantitative real-time PCR analyses of RASSF8 expression in eight paired primary ESCC tissues and matched ANT tissues. D., Kaplan-Meier curves of the overall survival of 137 ESCC patients with high or low RASSF8 expression. The $p$-value was computed by log-rank test. E., RASSF8 protein expression was lower in LN-positive (LN+) specimens than in LN-negative (LN-) specimens $(p=0.004)$. F., Representative staining images of RASSF8 and the lymphatic marker LYVE-1. Arrows indicate LYVE-1positive lymphatic vessels (left). Correlation of RASSF8 expression and lymphatic vessel density (LVD) was analyzed (right). Bars $=100$ $\mu \mathrm{m}$. IHC, immunohistochemistry. 


\section{RASSF8 downregulation increases VEGF-C expression in ESCC}

We investigated whether downregulating RASSF8 would increase the expression of VEGF-C, considered the most potent driver of lymphangiogenesis, in ESCC. Immunohistochemical staining of 23 ESCC primary tumors indicated that RASSF8 expression was inversely correlated with VEGF-C expression ( $p=0.014 ; \mathrm{R}=0.503$; Fig. 5A). Furthermore, protein expression of VEGF-C was downregulated in RASSF8 overexpression ESCC cell culture supernatants, but upregulated in RASSF8 knockdown ESCC cell culture supernatants (Fig. 5B). Accordingly, VEGF-C expression in the mice footpad tumors was significantly increased in RASSF8 knockdown tissues and was decreased in RASSF8 overexpression tissues (Fig.5C). As VEGF-C induces phosphorylation of the serine kinases AKT and ERK, which promotes HLEC proliferation, migration, and survival [19], we examined the effect of RASSF8-induced VEGF-C expression on AKT and ERK phosphorylation. Phosphorylated (phospho)-AKT (Ser 473) and phospho-ERK (T202/Y204) were dramatically upregulated in HLECs stimulated by TSNs from RASSF8-RNAi-transduced EC109 or KYSE410 cells (Fig. 5D). Importantly, we found that treatment with a PI3K/AKT signaling inhibitor LY294002 or MEK/ERK signaling inhibitor U0126 could reduce the ability of RASSF 8 knockdown cells to induce migration and tube formation in HLECs (Fig.5F and 5G). These results suggest that RASSF8 downregulation increases VEGF-C expression and promotes VEGF-C-induced signaling.
B

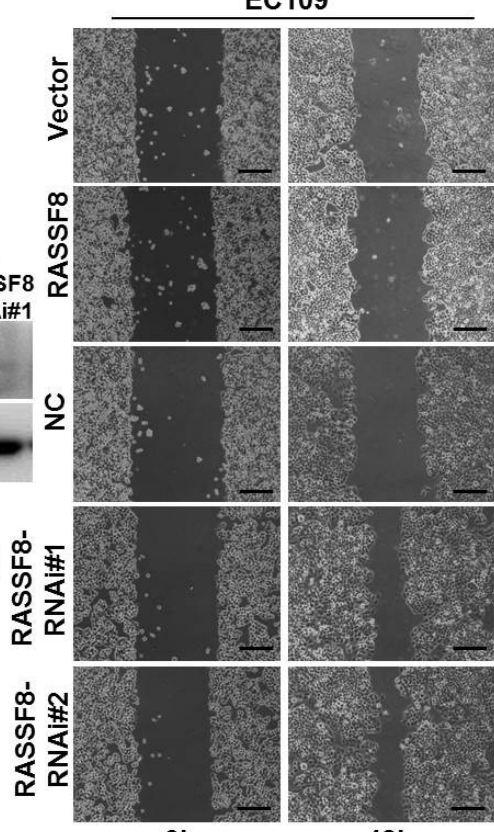

Oh

EC109

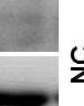

$48 h$
A

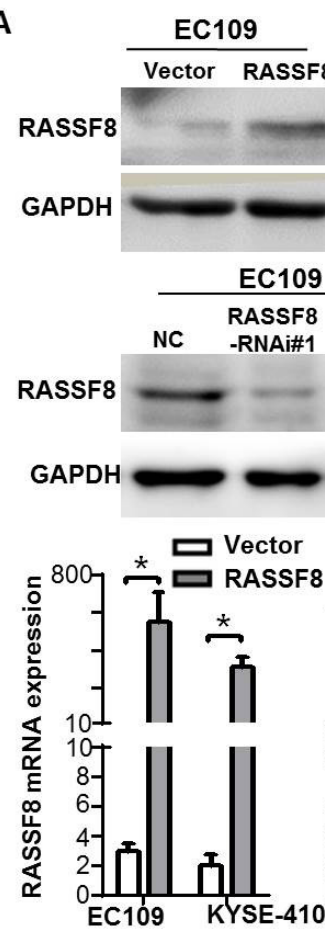

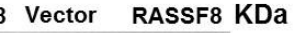

Vector

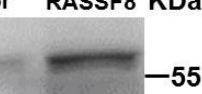

KYSE-410

\section{$-\infty$}

$-35$

YSE-410

RASSF8 RASSF8

-RNAi\#1 -RNAi\#1

C

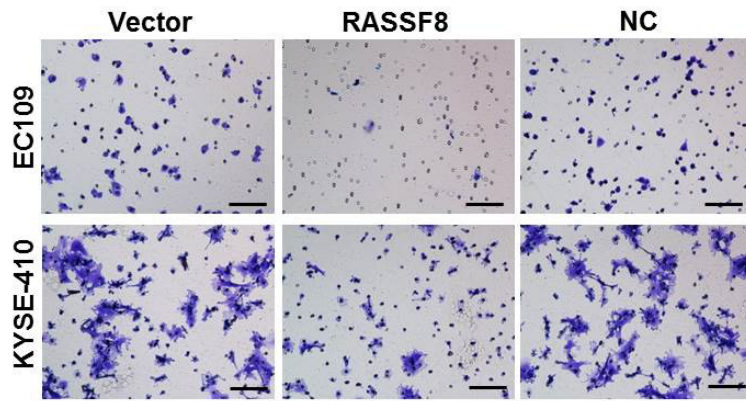

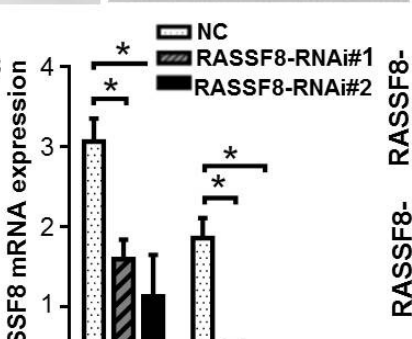

$\approx 0 \frac{1}{E C 109}$

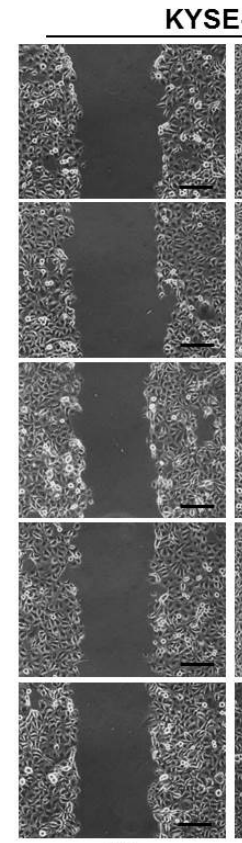

Oh
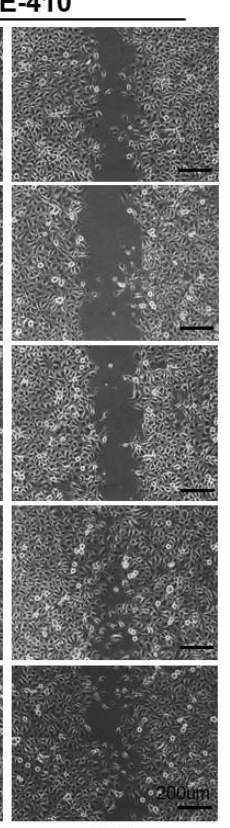

$24 h$

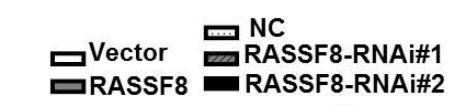

RASSF8-RNAi\#1 RASSF8-RNAi\#2
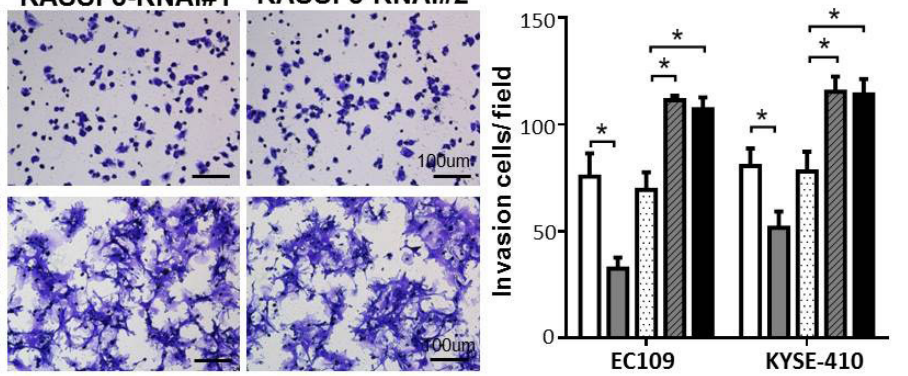

Figure 2: RASSF8 is essential for ESCC cell motility and invasiveness. A., Western blotting (top) and quantitative real-time PCR (bottom) analysis of RASSF8 overexpression and RASSF8 knockdown in EC109 and KYSE410 cell lines. B., Representative images of wound healing assay showing the motility of RASSF8 overexpression and knockdown cells as compared with the control cells. Bars $=$ $200 \mu \mathrm{m}$. C., Representative images of Transwell invasion assay showing ESCC cell invasion ability. Bars $=100 \mu \mathrm{m} .{ }^{*} p<0.05$ (Student's $t$-test). NC, negative control. 
A

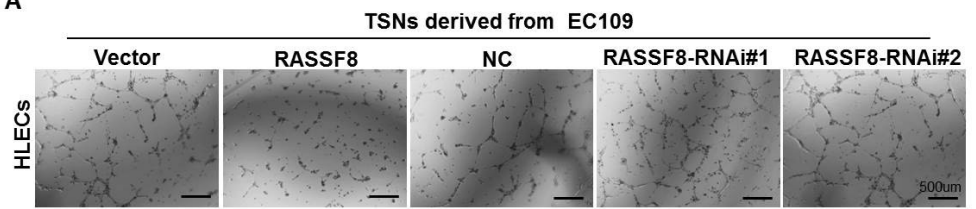

TSNs derived from KYSE-410-

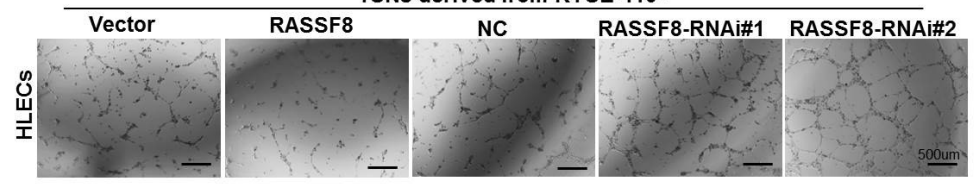

B

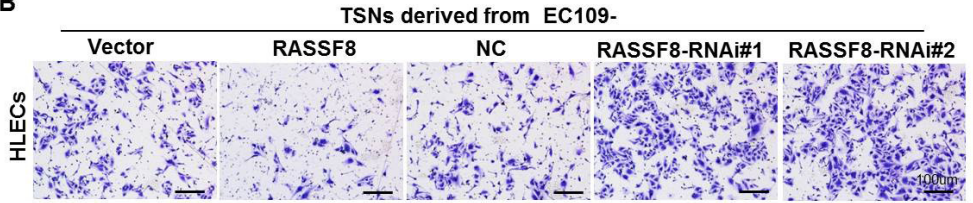

TSNs derived from KYSE-410.

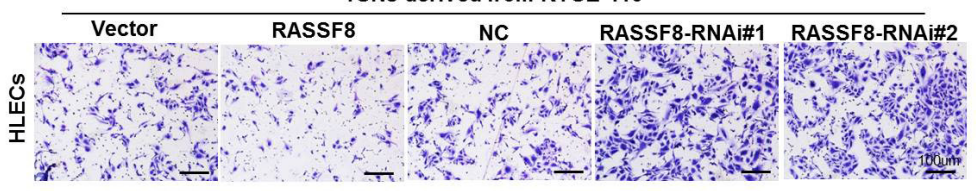

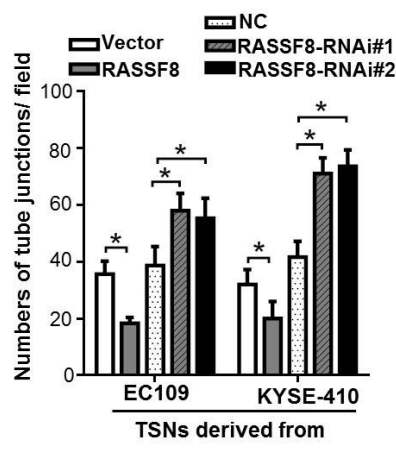

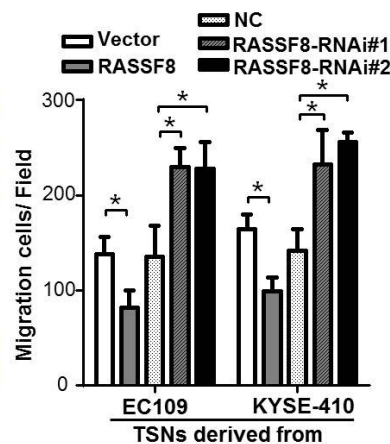

Figure 3: RASSF8 downregulation increases the ability of ESCC cells to induce lymphangiogenesis in vitro. A., Representative images (left) of HLECs cultured on Matrigel-coated plates with TSNs from ESCC cells. Bars $=500 \mu \mathrm{m}$. The average number of tube junctions per field was calculated (right). B., Representative images (left) and quantification (right) of HLEC migration assay. Bars $=100 \mu \mathrm{m}$. Data shown are the mean \pm SEM of three fields from three independent experiments. * $p<0.05$ (Student's $t$-test). $\mathrm{NC}$, negative control.

A
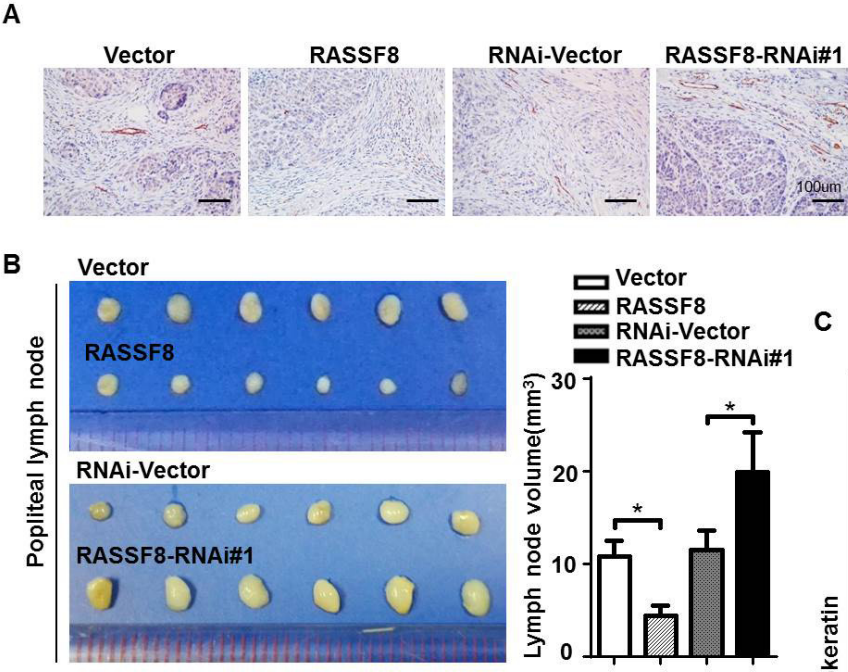

D Effect of RASSF8 expression on lymph node metastasis in vivo

\begin{tabular}{cccc}
\hline & $\begin{array}{c}\text { No.total } \\
\text { LNs }\end{array}$ & $\begin{array}{c}\text { No.metastasis } \\
\text { LNs }\end{array}$ & $\begin{array}{c}\text { Metastatic } \\
\text { Ratio } \\
(\%)\end{array}$ \\
vector & 6 & 4 & 66.7 \\
RASSF8 & 6 & 1 & 16.7 \\
RNAi-Vector & 6 & 3 & 50 \\
RASSF8-RNAi\#1 & 6 & 6 & 100 \\
\hline
\end{tabular}
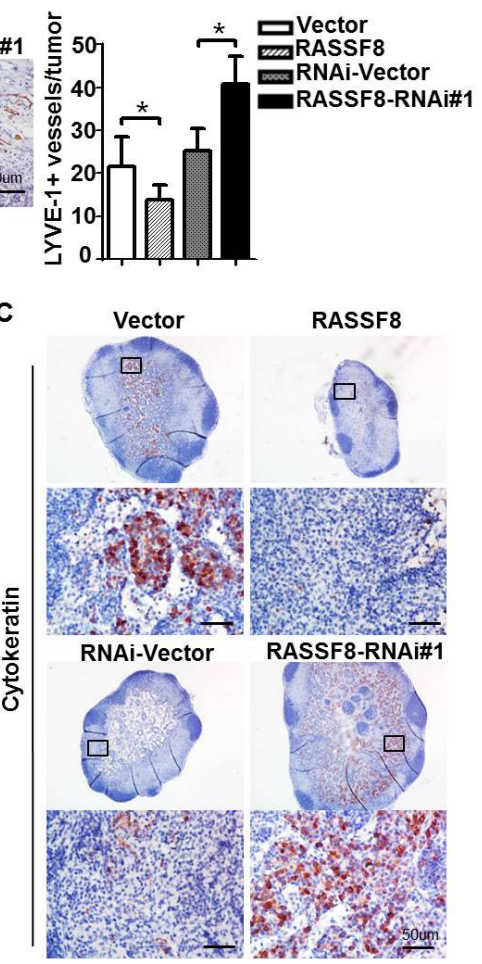

Figure 4: RASSF8 downregulation in ESCC cells promotes lymphangiogenesis and lymph node metastasis. A., AntiLYVE-1 antibody immunostaining of lymphatic vessels following ESCC cell inoculation in nude mice footpads. Shown are representative images (left) and quantitative analysis of peritumor lymphatic vessels (right). Bars $=100 \mu \mathrm{m}$. B., Representative images (left) and volumes (right) of popliteal lymph nodes. C., Representative images of anti-cytokeratin antibody-immunostained popliteal lymph nodes from mice inoculated with ESCC cells. Bars $=50 \mu \mathrm{m}$. D., Ratios of metastatic to total dissected popliteal lymph nodes from mice inoculated with ESCC cells. 
A

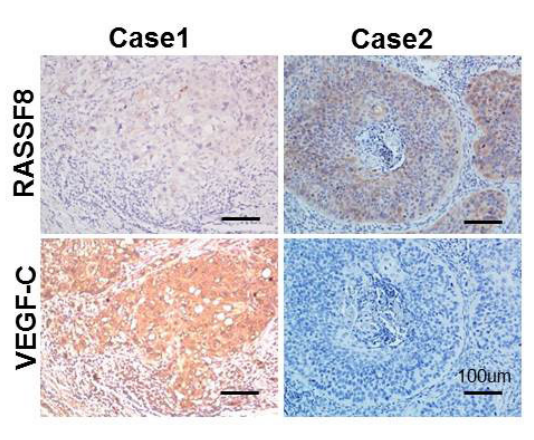

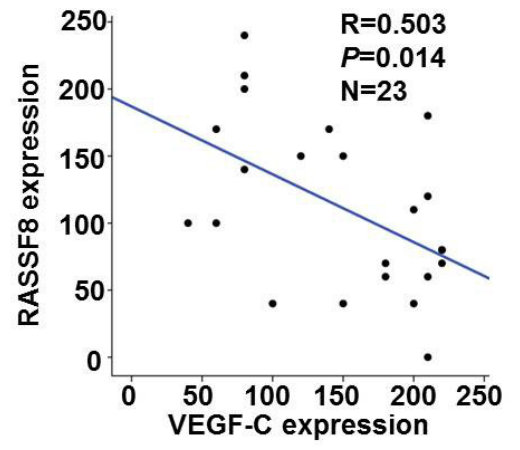

Vector $\mathrm{NC}$ $\begin{array}{ll}\text { 믐 } & \text { Rector } \\ \text { RASSSF8-RNAi\#1 } & \text { RASSF8-RNAi\#2 }\end{array}$

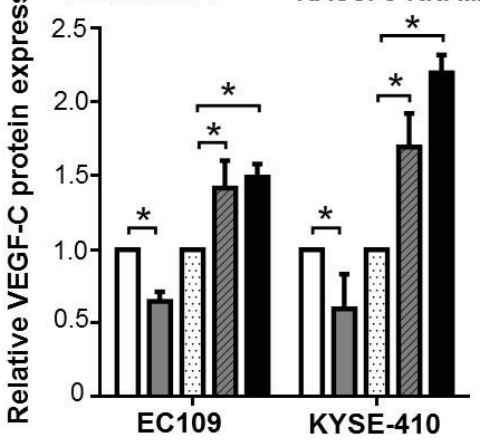

C

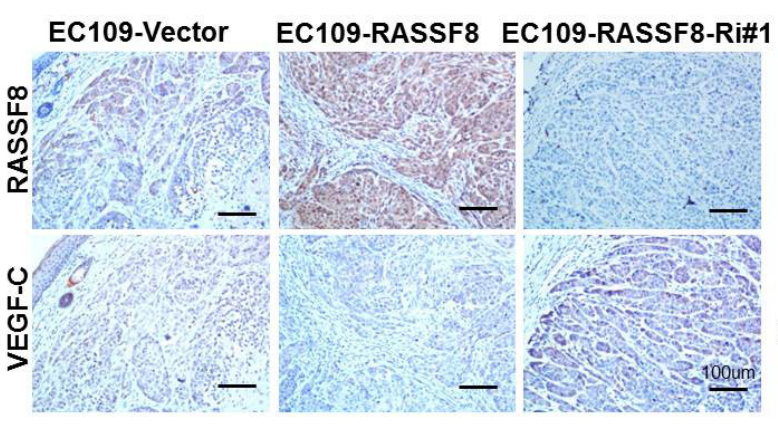

\section{D}

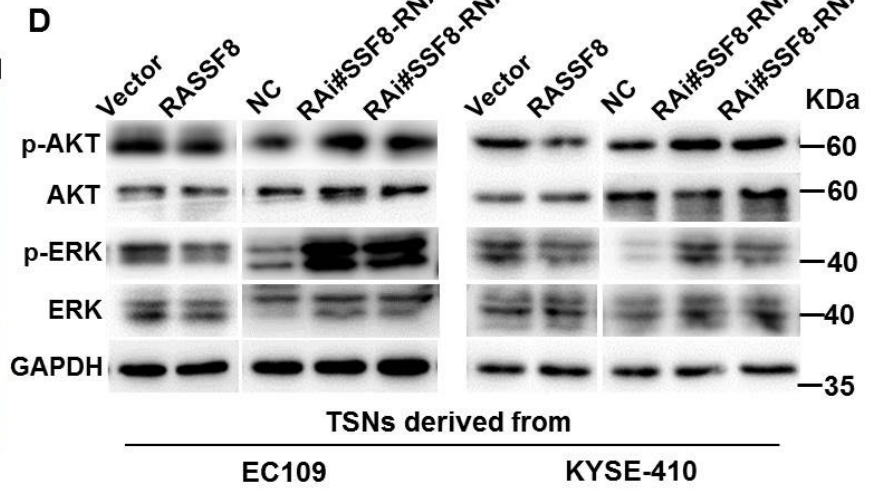

E

TSNs derived from EC109-RASSF8-RNAi\#1

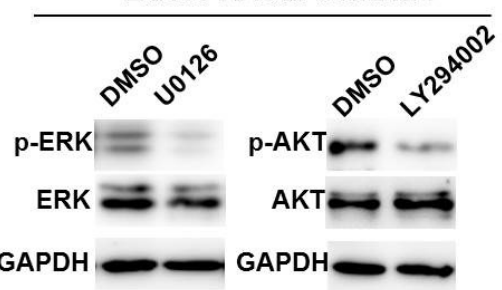

$\mathbf{F}$

\section{TSNs d}

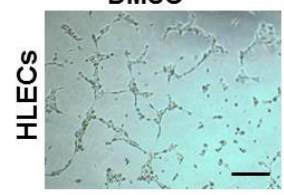

G

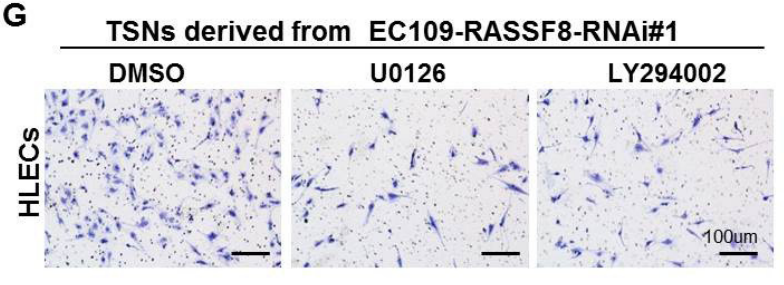

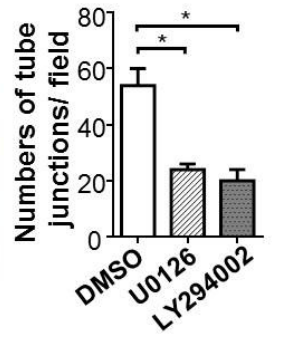

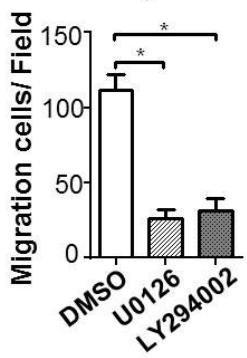

Figure 5: RASSF8 downregulates VEGF-C expression in ESCC cells. A., RASSF8 protein levels are inversely correlated with VEGF-C in clinical ESCC tissues $(n=23, p=0.014, \mathrm{R}=0.503)$. Left: Images of two representative cases. Right: Correlation analyses of RASSF8 and VEGF-C protein expression in 23 human ESCC tissues. Bars $=100 \mu \mathrm{m}$. B., ELISA of VEGF-C protein levels in the supernatants of the indicated cells. C., Immunohistochemical staining detection of VEGF-C protein levels in footpad tumors. Bars $=100 \mu \mathrm{m}$. D., Western blots of phospho-AKT (p-AKT, Ser 473) and phospho-ERK (p-ERK, T202/Y204) protein levels in TSN-treated human lymphatic endothelial cells (HLECs). E., Western blot analysis of phosphor-AKT (Ser374) and phosphor-ERK (T202/Y204) protein expression in HLECs cultured in TSNs derived from RASSF8 knockdown EC109 cells treated with U0126 or LY294002. F., G., Representative images and quantifications of matrigel tube formation $($ Bars $=500 \mu \mathrm{m})$ and transwell migration $($ Bars $=100 \mu \mathrm{m})$ assays, respectively, of HLECs cultured with TSN from the indicated cell. Error bars indicate the mean \pm SEM of three independent experiments. ${ }^{*} p<0.05$. 
RASSF8 downregulation enhances NF-kB transcriptional activity and causes subcellular redistribution of p65 in ESCC

We investigated whether RASSF8 is involved in pathway regulation of $\mathrm{NF}-\mathrm{\kappa B}$, a critical transcriptional regulator of VEGF-C $[20,21]$. We analyzed NF- $\mathrm{KB}$ transcriptional activity in RASSF8 overexpression and knockdown ESCC cells with a NF-kB luciferase report plasmid. RASSF8 downregulation significantly increased NF- $\kappa B$ transcriptional activity in both the EC109 and KYSE410 cell lines. By contrast, RASSF8 overexpression attenuated NF- $\mathrm{kB}$ transcriptional activity (Fig. 6A). P65 binds to $\kappa \mathrm{B}$ sites and transactivates a variety of genes to promote cellular transformation [22]; we examined the effect of RASSF8 on the subcellular distribution of

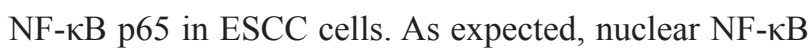
p65 expression was significantly decreased in RASSF8 overexpression cells and was increased in RASSF8 knockdown cells (Fig. 6B and 6C), suggesting that RASSF8 downregulation stimulate NF- $\kappa B$ by promoting NF- $\kappa B$ nuclear-cytoplasmic translocation.

\section{DISCUSSION}

Most epithelial cancers, including ESCC, initiate metastatic growth by disseminating through lymphatic vessels to the draining lymph nodes [4]. Examining metastatic cancer cells in the regional lymph nodes is a main prognostic factor of patient survival and determines the adjuvant therapies selected [3,23]. Cancer cells can use the lymphatic system to induce a conductive

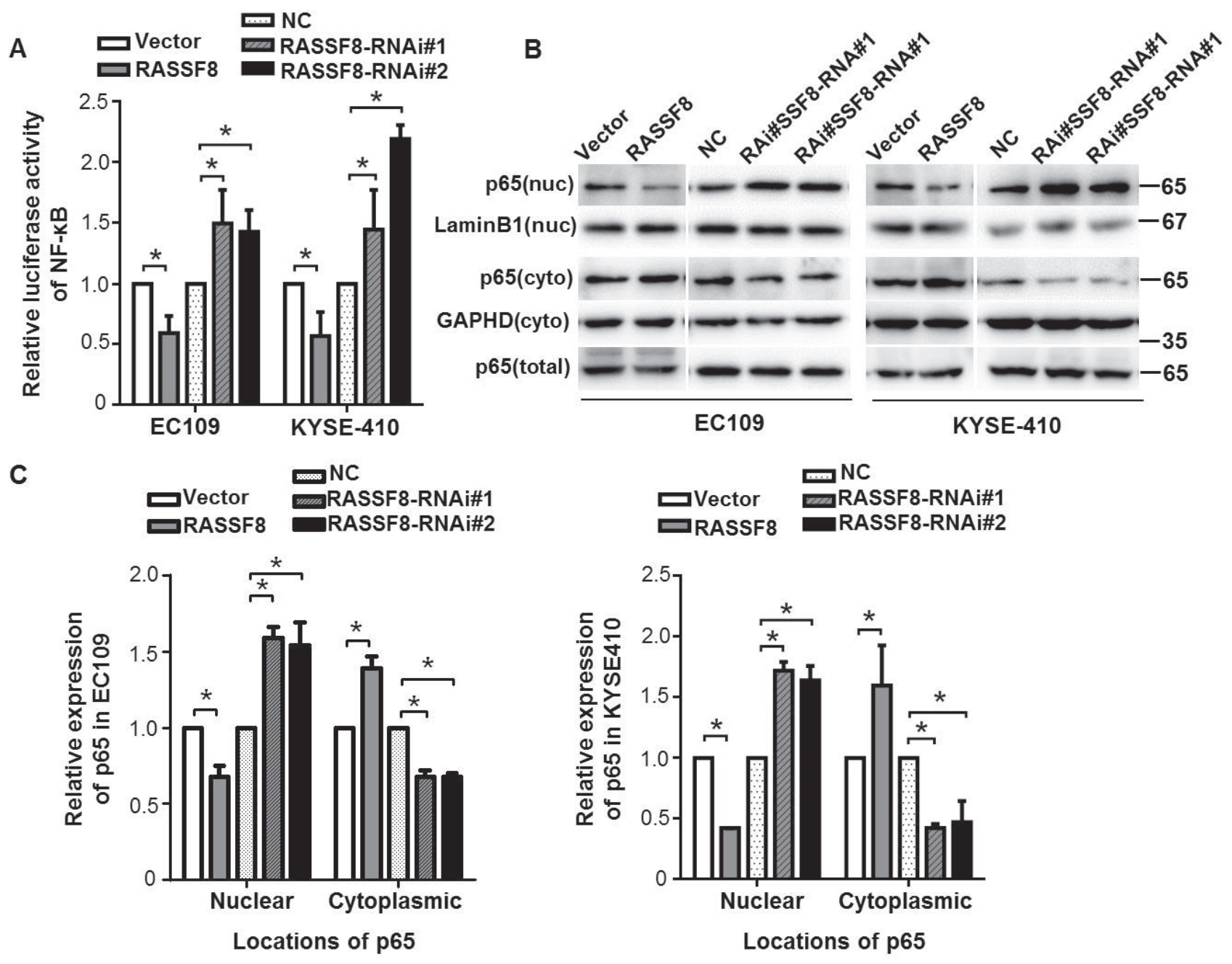

Figure 6: RASSF8 downregulation causes subcellular redistribution of p65 in ESCC cells. A., Relative expression of NF- $\kappa B$ luciferase reporter activities in the cells. Data represents percent change in normalized luciferase activity following RASSF8 or RASSF8RNAi co-transfection relative to the negative control (NC) vector \pm SEM from three independent experiments. ${ }^{*} p<0.05$. B., Western blots of nuclear (nuc), cytoplasmic (cyto), and total NF-кB p65 expression in the cells. Cytoplasmic control, GAPDH; nuclear control, lamin B1. C., Quantification analysis of subcellular distribution acquired from western blotting using ImageJ software (Wayne Rashband). Error bars indicate the mean \pm SEM from three independent exprements. ${ }^{*} p<0.05$. 
microenvironment for their survival in lymph nodes and at distant metastasis sites [24]. Developing new anti-cancer strategies by targeting the lymphatic system to reduce metastases and improve therapeutic efficiency would be desirable.

This report presents the first large-scale ESCC clinical study showing a significant clinical association between low RASSF8 expression and poor overall survival. RASSF8 downregulation in cancer cells contributes to increased ESCC cell invasion capacity, as RASSF8 re-expression inhibited cell invasion. Furthermore, loss of RASSF8 in ESCC cells may promote lymphatic endothelium migration and capillary-like tube formation, and in vivo tumor lymphangiogenesis and lymphatic metastasis, via VEGF-C upregulation under $\mathrm{NF}-\kappa \mathrm{B}$ transcriptional control.

VEGF-C and VEGF-D are the most potent regulatory growth factors of lymphatic endothelium proliferation, migration, and survival in human cancers $[25,26]$. VEGFR-3 activation induced by VEGF-C or VEGF-D leads to AKT and ERK phosphorylation, which promotes tumor-associated lymphatic vessel growth and enhances lymph node metastasis in many human cancers. Although fibroblast growth factor 2 (FGF-2) [27], insulinlike growth factor 1 (IGF-1) and IGF-2 [28], hepatocyte growth factor (HGF) [29], and platelet-derived growth factor-BB (PDGF-BB) [30] induce lymphangiogenesis in various contexts, most of these effects may be secondary to VEGF-C and VEGF-D induction in inflammatory cells and fibroblasts [31]. VEGF-C upregulation is the main mechanism by which oncogenes or other factors promote lymphangiogenesis and lymphatic metastasis in cancer. Our data suggest that RASSF8 acts on the lymphatic system if VEGF-C increases. The clinical results and in vitro/in vivo observations demonstrate higher VEGF-C expression and lymphatic vessel density in ESCC tumor tissues with loss of RASSF8. RASSF8 overexpression inhibits VEGF-C expression in ESCC cells, and AKT and ERK phosphorylation in the tumor-associated lymphatic endothelium.

$\mathrm{NF}-\kappa \mathrm{B}$, one of the major regulators of VEGF-C, is constitutively activated in ESCC and various cancers $[21,32,33]$. RASSF8 is a negative regulator of $N F-\kappa B$ transcriptional activity in lung cancer [17], where the regulatory protein I $\mathrm{I} B-\alpha$ is degraded and $\mathrm{p} 65$ dimers enter the nucleus following RASSF8 knockdown in A549 and H1792 cell lines. Our results are consistent with that report: in our study, p65 was upregulated in the nuclei of RASSF8 knockdown ESCC cells and downregulated in RASSF8 overexpression ESCC cells. Luciferase assay suggested that downregulated RASSF8 increases NF$\kappa \mathrm{B}$ activation. The observed $\mathrm{NF}-\kappa \mathrm{B}$ activation following RASSF8 depletion is the likely cause of the VEGF-C increase in ESCC cells.

The migration and invasion assays suggested that downregulated RASSF8 might also play a stimulatory role in cell motility, where cell motility was significantly decreased following RASSF8 depletion. RASSF8 colocalizes to the cell-cell adherens junction component $\beta$-catenin and binds to E-cadherin [17], a significant epithelial marker in epithelial-mesenchymal transition (EMT). Growing evidence from experimental and clinical studies demonstrate that EMT plays a critical role in tumor invasion and metastasis by endowing cells with a more motile, invasive phenotype [34]. Whether loss of RASSF8 contributes to EMT in ESCC still needs further investigation.

Nevertheless, the molecular mechanisms that lead to RASSF8 downregulation in ESCC have not been fully elucidated. The RASSF1A gene is one of the most widely reported epigenetically inactivated genes and could be a useful molecular marker for cancer diagnosis and prognosis [35]. RASSF2, RASSF4, RASSF5, and RASSF6 are also epigenetically inactivated in cancer [14]. However, it is unclear whether epigenetic modifications (i.e., promoter hypermethylation) cause RASSF8 downregulation in ESCC. The European Bioinformatics Institute $\mathrm{CpG}$ plot program predicts a 1201-bp CpG island upstream of, and inclusive of, the RASSF8 transcription start site. RASSF8 methylation has been reported in a small subset of leukemia [36]. We speculate that such epigenetic regulation of RASSF8 in ESCC may be possible. The exact mechanism of RASSF8 downregulation in ESCC requires intensive study.

In summary, we used clinical and experimental studies to establish a pro-metastasis role of downregulated RASSF8 in ESCCs. RASSF8 downregulation promoted ESCC metastasis by enhancing cell motility and invasiveness and by increasing lymphangiogenesis via the NF- $\kappa \mathrm{B} / \mathrm{VEGF}-\mathrm{C}$ axis. Our characterization of the functions and mechanism of downregulated RASSF8 will not only increase our understanding of ESCC metastasis, but also presents a new therapeutic target for ESCC treatment.

\section{MATERIALS AND METHODS}

\section{Ethics statement}

This investigation has been conducted in accordance with ethical standards and according to the Declaration of Helsinki and to national and international guidelines, and has been approved by Sun Yat-sen University Medical Ethics Committee.

\section{Patient information and tissue samples}

We enrolled 137 patients diagnosed with ESCC who went esophagectomy and lymphadenectomy at the Sun Yat-sen University Cancer Center from 2002 to 
2009. Patients attended follow-up visits regularly; written informed consent was obtained from all patients prior to the study. Two experienced pathologists performed the grading and histopathological subtyping of specimens. Eight fresh ESCC and paired ANT esophageal tissues were collected for quantitative real-time PCR. Table 1 summarizes the clinical information of the samples.

\section{RNA isolation and quantitative real-time PCR}

Total RNA was isolated from cells and tissues using TRIzol reagent (Life Technologies, Carlsbad, CA, USA) according to the manufacturer's instructions. Reverse transcriptase reactions using MMLV reverse transcriptase reagents (Promega, Madison, WI, USA) were performed according to the manufacturer's protocol. Quantitative real-time PCR was performed using GoTaq qPCR Master Mix (Promega) in a Roche LightCycler 480 II PCR system (Roche Diagnostics, Rotkreuz, Switzerland). Relative gene expression was presented as comparative threshold cycle $\left(2^{-\Delta \Delta \mathrm{Ct}}\right)$ values and was representative of at least three independent experiments. Glyceraldehyde-3-phosphate dehydrogenase (GAPDH) and $\beta$-actin were used to normalize target gene expression. The reverse transcription-PCR primer sequences are as follows: RASSF8, forward 5'-AAGTATGGGTGGATGGAGTTCAG-3' and reverse 5'-ATGAGGTGCTAAGTGTCTTTCAG-3'; GAPDH, forward 5'-CTCCTCCTGTTCGACAGTCAGC-3' and reverse 5'-CCCAATACGACCAAATCCGTT-3'; $\beta$-actin, forward 5'-TGGCACCCAGCACAATGAA-3' and reverse 5'-CTAAGTCATAGTCCGCCTAGAAGCA-3'; VEGF-C, forward 5'-CGGACTCGACCTCTCGG-3' and reverse 5'-TGGACACAGACCGTAACTGC-3'.

\section{Immunohistochemistry analysis}

Paraffin-embedded, formalin-fixed samples were cut into $4-\mu \mathrm{m}$ sections. Immunohistochemical staining of ESCC tissue sections was performed using diaminobenzidine (Dako, Copenhagen, Denmark) as the chromogen. Serial sections were labeled with antibodies against RASSF8 (Sigma-Aldrich, St. Louis, MO, USA, HPA038163) and LYVE-1 (Abcam, Cambridge, UK, ab14917), or VEGF-C (R\&D System, Inc, Minneapolis, MN55413, USA, AF752). We used an immunohistological reactivity scoring system (H-score) $[37,38]$ to determine RASSF8 and VEGF-C protein levels in tissue. The $\mathrm{H}$-score takes into account the percentage of positivestaining cells $(0-100 \%)$ in each staining intensity category ( 0 to $3+$ ) and computes a final score. The H-score is obtained as follows: $(3 \times$ percentage of strong staining $)$ $+(2 \times$ percentage of moderate staining $)+$ (percentage of weak staining), yielding an $\mathrm{H}$-score range of 0 to 300 . The tissues were divided into high and low RASSF8 expression based on the median value of RASSF8 expression level per slide (Supplementary Fig. 2). The average number of LYVE-1-positive stained lymphatic vessels was counted in two selected vessel hot spots at $\times 200$ magnification. The tissues were divided into high and low lymphatic vessel density based on the average number of LYVE-1-positive stained lymphatic vessels per $0.25 \mathrm{~mm}^{2}$.

\section{Cell lines}

The ESCC cell lines KYSE30, KYSE140, KYSE180, KYSE410, KYSE510, KYSE520 were obtained from Deutsche Sammlung von Mikroorganismen und Zellkulturen (DSMZ), the German Resource Center for Biological Material[39]. EC18, and EC109, and the immortalized esophageal epithelial cell line NE1 were kept in the State Key Laboratory of Oncology in South China, Sun Yat-sen University Cancer Center. The cell lines were cultured in Dulbecco's modified Eagle's medium (DMEM; Life Technologies) supplemented with $10 \%$ fetal bovine serum (FBS; Life Technologies) in a 5\% $\mathrm{CO}_{2}$ atmosphere at $37^{\circ} \mathrm{C}$. HLECs were purchased from ScienCell Research Laboratories (Carlsbad, CA, USA) and cultured in endothelial cell medium (ECM; ScienCell Research Laboratories) with 5\% FBS and endothelial growth medium supplements (VEGF-C-free).

\section{Plasmid, transfection, and TSNs}

A pcDNA3.1/RASSF8 construct was generated by subcloning the PCR-amplified full-length human RASSF8 coding sequence into a pcDNA3.1 plasmid. The RASSF8-targeting small interfering RNA (siRNA) sequences are as follows: RNAi\#1, forward 5'-CUGGAAGGUACACCCUUAUTT-3', reverse 5'-AUAAGGGUGUACCUUCCAGTT-3'; RNAi\#2, forward, 5'-GGCUCGAAUUCCUGAAAGATT-3', reverse 5'-UCUUUCAGGAAUUCGAGCCTT-3'. The NF- $\kappa$ B luciferase report plasmid was a gift from Linbai Ye (College of Life Science, Wuhan University, Wuhan, China)[40, 41]. SiRNA or plasmid transfection was conducted using Lipofectamine 2000 (Invitrogen) according to the manufacturer's instruction. At $48 \mathrm{~h}$ after transfection, the TSNs were harvested, centrifuged, and stored in aliquots at $-80^{\circ} \mathrm{C}$. Stable cell lines expressing RASSF8 or RASSF8-RNAi were selected with puromycin for 10 days.

\section{Western blotting assay}

Protein was extracted using a protein extraction kit (KeyGen Biotech, KGP250-2100, Nanjing, China) according to the manufacturer's instructions. The primary antibodies used were anti-RASSF8 (Sigma, HPA038163), 
anti-VEGF-C (R\&D, AF752), anti-phospho-AKT (Cell Signaling, Danvers, MA, USA, 4691S), anti-AKT (Cell Signaling, 4060S), anti-phospho-ERK1/2 (Cell Signaling, 4370P), anti-ERK (Cell Signaling, 9102S), anti-p65 (Cell Signaling, 6956S), anti- $\beta$-tubulin (Cell Signaling, 2128S), and anti-GAPDH (Abgent, San Diego, CA, USA). Antilamin B1 (KeyGen Biotech, KGAA004-2) was used as the control nuclear protein. Signals were detected using Immobilon Western Chemiluminescent HRP Substrate (Millipore, Billerica, MA, USA) and a Tanon 5200 Luminescent Imaging Workstation (Tanon, Shanghai, China).

\section{Subcellular fractionation}

RASSF8 overexpressing and knockdown cells were subjected to subcellular fractionation using the cytoplasmic and nuclear protein extraction kit (KeyGen Biotech, KGP150), according to the manufacturer's instructions. The efficacy of fractionation was determined via western blotting by stripping the polyvinylidene fluoride membranes and re-probed with LaminB1 as the nuclear control protein and GAPDH antibodies as the cytosolic control. The experiment was performed in triplicate.

\section{Wound healing assay}

In $95-100 \%$ confluent ESCC cultures, we generated wounds with a pipette tip, and obtained images of the wound healing (migration) at the $0 \mathrm{~h}, 24 \mathrm{~h}$ and $48 \mathrm{~h}$.

\section{Invasion assay}

BD Falcon Cell Culture Inserts with $8.0-\mu \mathrm{m}$ pores (BD Biosciences, Franklin Lakes, NJ, USA) pre-coated with Matrigel (BD Biosciences) were placed in a 24well plate containing $600 \mu 1 \quad 10 \%$ FBS/DMEM in the lower chamber. ESCC cells were placed in the upper chamber and incubated at $37^{\circ} \mathrm{C}$ for $24 \mathrm{~h}$. Cells that migrated to the lower membrane surface were fixed in $1 \%$ paraformaldehyde, stained with crystal violet and counted under an optical microscope (× 200 magnification $)$.

\section{Migration assay}

Migration assay was performed using a Transwell chamber (BD Biosciences). HLECs $\left(2 \times 10^{4}\right)$ in serumfree medium were placed in the upper chamber. The lower chambers were filled with the ESCC TSNs. After 10-h incubation, HLECs that passed through the membrane were fixed and stained with crystal violet. The number of cells was counted under a microscope $(\times 200$ magnification) from three randomly chosen fields per membrane, and the average number of cells per field was calculated.

\section{Tube formation assay}

For capillary-like tube formation assays, $75 \mu \mathrm{l} /$ well Matrigel and $75 \mu \mathrm{l} /$ well serum-free ECM were mixed and polymerized in a 48 -well plate. Then, $2 \times 10^{4}$ HLECs in $100 \mu \mathrm{l} \mathrm{ECM}$ and $400 \mu \mathrm{l} \mathrm{TSNs}$ were incubated on the Matrigel layer in each well. After $4 \mathrm{~h}$, images of the tube formation were obtained using phase-contrast microscopy at $\times 40$ magnification.

\section{Animal model}

BALB/c-nu mice (4-5 weeks old) were purchased from the Guangzhou University of Chinese Medicine Experimental Animal Center (Guangzhou, China). EC109 cells infected with lentivirus RASSF8, RASSF8-RNAi, or vector, negative control were suspended in sterile phosphate-buffered saline with 25\% Matrigel and injected into the lower mammary fat pads of the mice (four groups, $n=6$ per group). After 28 days, the tumors and popliteal lymph nodes were excised and measured. Serial 4.0- $\mu \mathrm{m}$ sections were obtained and analyzed by immunochemical staining with anti-LYVE-1 and anti-cytokeratin antibodies (MXB, Fuzhou, China). The popliteal lymph nodes were estimated using the equation $\left(\mathrm{L} \times \mathrm{W}^{2}\right) / 2$. The ratios of positive-stained lymph nodes to total lymph nodes were calculated.

\section{Enzyme-linked immunosorbent assay}

The concentration of VEGF-C was determined by a commercially available VEGF-C ELISA Kit (Cloud-Clone Corp, Houston, TX, USA). Enzyme-linked immunosorbent assay was performed according to the instructions of the manufacturer. Briefly, the cell culture supernatants were added to wells coated with VEGF-C polyclonal antibody, and incubated with biotinylated monoclonal anti-VEGF-C antibody at $37^{\circ} \mathrm{C}$ for 24 hours. Horseradish peroxidase was used to catalyze the color development, and terminated with stop solution. Absorbance was measured at 450nm. The protein concentrations were determined relative to standard samples.

\section{Inhibiting signaling assays}

The inhibitors of PI3K/AKT signaling LY294002 (Selleck Chemicals, Houston, TX, USA) and of MEK/ ERK signaling U0126 (Selleck Chemicals) were dissolved in DMSO respectively at a stock concentration of $10 \mathrm{mM}$ and added to cell cultures at a concentration of $10 \mathrm{uM}$. After 24 hours, cell culture supernatants were collected 
for the HLECs incubation and the transwell migration and matrigel tube formation assays.

\section{Luciferase reporter assay}

Briefly, $2 \times 10^{4}$ ESCC cells were seeded in triplicate in 24-well plates and allowed to settle for $12 \mathrm{~h}$. Cells were transfected with $200 \mathrm{ng} N \mathrm{NF}-\kappa \mathrm{B}$ luciferase reporter plasmid and $10 \mathrm{ng}$ pRL-TK Renilla plasmid (Promega) using Lipofectamine 2000. At $24 \mathrm{~h}$ after transfection, cells were harvested and luciferase activity was analyzed using a Dual-Luciferase Reporter Assay System (Promega) according to the manufacturer's instructions. Firefly luciferase activity was normalized to that of Renilla luciferase as the transfection control.

\section{Statistical analysis}

Statistical analysis was performed using SPSS Standard version 16.0 (SPSS Inc, Chicago, IL, USA). Data are presented as the mean \pm standard error of the mean (SEM). Overall survival was defined as the period between the date of surgery to disease-related death. Data were censored at the last follow-up for patients who were disease-free or alive at the time of analysis. Survival functions were examined by Kaplan-Meier analysis. Cox regression analysis was used to assess factors related to survival. Correlation coefficients were computed to measure the degree of association between biomarkers. Data from cell line experiments were assessed by a twotailed Student's $t$-test. $P$-values $<0.05$ were considered significant.

\section{ACKNOWLEDGMENTS}

This research was supported by grants from the National Natural Science Foundation of China (Grant numbers 81171948, 81372275), the Key Program of the Natural Science Foundation of Guangdong Province, China (Grant number S2012020011060), the Project of State Key Laboratory of Oncology in South China (Grant number 030041060004), the Project of Guangzhou science and technology plan (2014J4100238) to Min Zheng, and supported in part by the Natural Science Foundation of Guangdong Province, China (Grant number S2013010011548) to Jian-Hua Wang.

\section{CONFLICTS OF INTEREST}

The authors declare that there are no conflicts of interest.

\section{REFERENCES}

1. Jemal A, Bray F, Center MM, Ferlay J, Ward E and Forman D. Global cancer statistics. CA: a cancer journal for clinicians. 2011; 61:69-90.

2. Enzinger PC and Mayer RJ. Esophageal cancer. The New England journal of medicine. 2003; 349:2241-2252.

3. Hosch SB, Stoecklein NH, Pichlmeier U, Rehders A, Scheunemann P, Niendorf A, Knoefel WT and Izbicki JR. Esophageal cancer: the mode of lymphatic tumor cell spread and its prognostic significance. Journal of clinical oncology : official journal of the American Society of Clinical Oncology. 2001; 19:1970-1975.

4. Karaman S and Detmar M. Mechanisms of lymphatic metastasis. The Journal of clinical investigation. 2014; 124:922-928.

5. Zheng W, Aspelund A and Alitalo K. Lymphangiogenic factors, mechanisms, and applications. The Journal of clinical investigation. 2014; 124:878-887.

6. Karkkainen MJ, Haiko P, Sainio K, Partanen J, Taipale J, Petrova TV, Jeltsch M, Jackson DG, Talikka M, Rauvala $\mathrm{H}$, Betsholtz $\mathrm{C}$ and Alitalo K. Vascular endothelial growth factor $\mathrm{C}$ is required for sprouting of the first lymphatic vessels from embryonic veins. Nature immunology. 2004; 5:74-80.

7. Ding MX, Lin XQ, Fu XY, Zhang N and Li JC. Expression of vascular endothelial growth factor-C and angiogenesis in esophageal squamous cell carcinoma. World journal of gastroenterology : WJG. 2006; 12:4582-4585.

8. Kitadai Y, Amioka T, Haruma K, Tanaka S, Yoshihara M, Sumii K, Matsutani N, Yasui W and Chayama K. Clinicopathological significance of vascular endothelial growth factor (VEGF)-C in human esophageal squamous cell carcinomas. International journal of cancer Journal international du cancer. 2001; 93:662-666.

9. Makinen T, Veikkola T, Mustjoki S, Karpanen T, Catimel B, Nice EC, Wise L, Mercer A, Kowalski H, Kerjaschki D, Stacker SA, Achen MG and Alitalo K. Isolated lymphatic endothelial cells transduce growth, survival and migratory signals via the VEGF-C/D receptor VEGFR-3. The EMBO journal. 2001; 20:4762-4773.

10. Shimizu K, Kubo H, Yamaguchi K, Kawashima K, Ueda Y, Matsuo K, Awane M, Shimahara Y, Takabayashi A, Yamaoka Y and Satoh S. Suppression of VEGFR-3 signaling inhibits lymph node metastasis in gastric cancer. Cancer science. 2004; 95:328-333.

11. He Y, Kozaki K, Karpanen T, Koshikawa K, Yla-Herttuala $\mathrm{S}$, Takahashi $\mathrm{T}$ and Alitalo K. Suppression of tumor lymphangiogenesis and lymph node metastasis by blocking vascular endothelial growth factor receptor 3 signaling. Journal of the National Cancer Institute. 2002; 94:819-825.

12. Lin J, Lalani AS, Harding TC, Gonzalez M, Wu WW, Luan B, Tu GH, Koprivnikar K, VanRoey MJ, He Y, Alitalo K and Jooss K. Inhibition of lymphogenous metastasis using 
adeno-associated virus-mediated gene transfer of a soluble VEGFR-3 decoy receptor. Cancer research. 2005; 65:69016909.

13. Liu L, Lin C, Liang W, Wu S, Liu A, Wu J, Zhang X, Ren P, Li M and Song L. TBL1XR1 promotes lymphangiogenesis and lymphatic metastasis in esophageal squamous cell carcinoma. Gut. 2015; 64:26-36.

14. Underhill-Day N, Hill V and Latif F. N-terminal RASSF family: RASSF7-RASSF10. Epigenetics : official journal of the DNA Methylation Society. 2011; 6:284-292.

15. Sherwood V, Recino A, Jeffries A, Ward A and Chalmers AD. The N-terminal RASSF family: a new group of Rasassociation-domain-containing proteins, with emerging links to cancer formation. The Biochemical journal. 2010; 425:303-311.

16. Scheel $\mathrm{H}$ and Hofmann K. A novel interaction motif, SARAH, connects three classes of tumor suppressor. Current biology : CB. 2003; 13:R899-900.

17. Lock FE, Underhill-Day N, Dunwell T, Matallanas D, Cooper W, Hesson L, Recino A, Ward A, Pavlova T, Zabarovsky E, Grant MM, Maher ER, Chalmers AD, Kolch $\mathrm{W}$ and Latif F. The RASSF8 candidate tumor suppressor inhibits cell growth and regulates the Wnt and NF-kappaB signaling pathways. Oncogene. 2010; 29:4307-4316.

18. Falvella FS, Manenti G, Spinola M, Pignatiello C, Conti B, Pastorino U and Dragani TA. Identification of RASSF8 as a candidate lung tumor suppressor gene. Oncogene. 2006; 25:3934-3938.

19. Salameh A, Galvagni F, Bardelli M, Bussolino F and Oliviero S. Direct recruitment of CRK and GRB2 to VEGFR-3 induces proliferation, migration, and survival of endothelial cells through the activation of ERK, AKT, and JNK pathways. Blood. 2005; 106:3423-3431.

20. Karin M and Greten FR. NF-kappaB: linking inflammation and immunity to cancer development and progression. Nature reviews Immunology. 2005; 5:749-759.

21. Lin C, Song L, Gong H, Liu A, Lin X, Wu J, Li M and Li J. Nkx2-8 downregulation promotes angiogenesis and activates NF-kappaB in esophageal cancer. Cancer research. 2013; 73:3638-3648.

22. Hayden MS and Ghosh S. Shared principles in NF-kappaB signaling. Cell. 2008; 132:344-362.

23. Lyu X, Huang J, Mao Y, Liu Y, Feng Q, Shao K, Gao S, Jiang Y, Wang J and He J. Adjuvant chemotherapy after esophagectomy: is there a role in the treatment of the lymph node positive thoracic esophageal squamous cell carcinoma? Journal of surgical oncology. 2014; 110:864868.

24. Alitalo A and Detmar M. Interaction of tumor cells and lymphatic vessels in cancer progression. Oncogene. 2012; 31:4499-4508.

25. Noguchi T, Takeno S, Shibata T, Uchida Y, Yokoyama $\mathrm{S}$ and Muller W. VEGF-C expression correlates with histological differentiation and metastasis in squamous cell carcinoma of the esophagus. Oncology reports. 2002; 9:995-999.

26. Mohammed RA, Green A, El-Shikh S, Paish EC, Ellis IO and Martin SG. Prognostic significance of vascular endothelial cell growth factors -A, -C and -D in breast cancer and their relationship with angio- and lymphangiogenesis. British journal of cancer. 2007; 96:1092-1100.

27. Chang LK, Garcia-Cardena G, Farnebo F, Fannon M, Chen EJ, Butterfield C, Moses MA, Mulligan RC, Folkman J and Kaipainen A. Dose-dependent response of FGF-2 for lymphangiogenesis. Proceedings of the National Academy of Sciences of the United States of America. 2004; 101:11658-11663.

28. Bjorndahl M, Cao R, Nissen LJ, Clasper S, Johnson LA, Xue Y, Zhou Z, Jackson D, Hansen AJ and Cao Y. Insulinlike growth factors 1 and 2 induce lymphangiogenesis in vivo. Proceedings of the National Academy of Sciences of the United States of America. 2005; 102:15593-15598.

29. Kajiya K, Hirakawa S, Ma B, Drinnenberg I and Detmar M. Hepatocyte growth factor promotes lymphatic vessel formation and function. The EMBO journal. 2005; 24:28852895.

30. Cao R, Bjorndahl MA, Religa P, Clasper S, Garvin S, Galter D, Meister B, Ikomi F, Tritsaris K, Dissing S, Ohhashi T, Jackson DG and Cao Y. PDGF-BB induces intratumoral lymphangiogenesis and promotes lymphatic metastasis. Cancer cell. 2004; 6:333-345.

31. Karpanen T and Alitalo K. Molecular biology and pathology of lymphangiogenesis. Annual review of pathology. 2008; 3:367-397.

32. Grivennikov SI, Greten FR and Karin M. Immunity, inflammation, and cancer. Cell. 2010; 140:883-899.

33. Tsai PW, Shiah SG, Lin MT, Wu CW and Kuo ML. Upregulation of vascular endothelial growth factor $\mathrm{C}$ in breast cancer cells by heregulin-beta 1 . A critical role of p38/ nuclear factor-kappa B signaling pathway. The Journal of biological chemistry. 2003; 278:5750-5759.

34. Thiery JP and Sleeman JP. Complex networks orchestrate epithelial-mesenchymal transitions. Nature reviews Molecular cell biology. 2006; 7:131-142.

35. Agathanggelou A, Cooper WN and Latif F. Role of the Ras-association domain family 1 tumor suppressor gene in human cancers. Cancer research. 2005; 65:3497-3508.

36. Hesson LB, Dunwell TL, Cooper WN, Catchpoole D, Brini AT, Chiaramonte R, Griffiths M, Chalmers AD, Maher ER and Latif F. The novel RASSF6 and RASSF10 candidate tumour suppressor genes are frequently epigenetically inactivated in childhood leukaemias. Molecular cancer. 2009; 8:42.

37. Gombos Z, Xu X, Chu CS, Zhang PJ and Acs G. Peritumoral lymphatic vessel density and vascular endothelial growth factor $\mathrm{C}$ expression in early-stage squamous cell carcinoma of the uterine cervix. Clinical cancer research : an official 
journal of the American Association for Cancer Research. 2005; 11:8364-8371.

38. Pirker R, Pereira JR, von Pawel J, Krzakowski M, Ramlau R, Park K, de Marinis F, Eberhardt WE, Paz-Ares L, Storkel S, Schumacher KM, von Heydebreck A, Celik I and O'Byrne KJ. EGFR expression as a predictor of survival for first-line chemotherapy plus cetuximab in patients with advanced non-small-cell lung cancer: analysis of data from the phase 3 FLEX study. The Lancet Oncology. 2012; 13:33-42.

39. Shimada $Y$, Imamura $M$, Wagata $T$, Yamaguchi $N$ and Tobe T. Characterization of 21 newly established esophageal cancer cell lines. Cancer. 1992; 69:277-284.

40. Zhang Y, Li Y, Ma Y, Liu S, She Y, Zhao P, Jing M, Han T, Yan C, Wu Z, Gao J and Ye L. Dual effects of interleukin-18: inhibiting hepatitis $\mathrm{B}$ virus replication in HepG2.2.15 cells and promoting hepatoma cells metastasis. American journal of physiology Gastrointestinal and liver physiology. 2011; 301:G565-573.

41. Gao S, Song L, Li J, Zhang Z, Peng H, Jiang W, Wang Q, Kang $\mathrm{T}$, Chen $\mathrm{S}$ and Huang W. Influenza A virus-encoded NS1 virulence factor protein inhibits innate immune response by targeting IKK. Cellular microbiology. 2012; 14:1849-1866. 